\title{
POLARBEAR-2 receiver system on the Simons Array telescopes for CMB polarization measurements
}

\author{
Yasuto Hori* for the POLARBEAR-2 and Simons Array Collaborations \\ High Energy Accelerator Research Organization (KEK) \\ E-mail: yhori@post.kek.jp
}

\begin{abstract}
The POLARBEAR-2 (PB-2) and Simons Array are upgrade projects of POLARBEAR-1 experiment. They are ground-based Cosmic Microwave Background (CMB) polarization experiments at Atacama in Chile. PB-2, the first newly designed receiver on the one of the Simons Array telescopes, will be deployed in 2015. The PB-2 receiver has 1,897 lenslet-coupled pixels on the focal plane, each of which is dual-polarization and dual frequency $(95 / 150 \mathrm{GHz})$ with total of 7.588 Transition Edge Sensors (TES) bolometric detectors. The expected noise equivalent temperature of the PB-2 receiver is $5.8 \mu \mathrm{K} \sqrt{s}$ for $95 \mathrm{GHz}$ and $150 \mathrm{GHz}$. The PB-2 has advantages of high sensitivity, high angular resolution, dual frequency observation, accessibility to a large sky and cost-efficient scalability to the full Simons Array project. These features allow us to investigate the inflationary and lensing CMB B-mode polarization in detail as a next generation experiment.
\end{abstract}

Technology and Instrumentation in Particle Physics 2014,

2-6 June, 2014

Amsterdam, the Netherlands

${ }^{*}$ Speaker. 


\section{Introduction}

Recently, the BICEP2 experiment reported the detection of the CMB B-mode polarization angular power spectrum at large scale as shown by the green points in Figure 1 [1]. This detection is consistent with the peak signal at $l \sim 90$ which can be generated by the inflation model with the tensor-to-scalar ratio of $r=0.2$ in the early universe although there exists some uncertainty of the foreground contributions. The tensor-to-scalar ratio of $r=0.2$ corresponds to the Grand Unified Theory (GUT) energy scale $\left(10^{16} \mathrm{GeV}\right)$. This discovery provides an extraordinary opportunity to investigate inflation models in detail, providing insight to the very high energy physics which is impossible to access by ordinary accelerator experiments.

Just before the BICEP2 detection, the POLARBEAR-1 (PB-1) experiment announced the result of CMB B-mode polarization power spectrum at small scale $(l \sim 1000)$ as shown by the red points in Figure 1 [2]. It is consistent with the CMB B-mode polarization power spectrum generated by the gravitational lensing effect on the CMB E-mode polarization. Precise measurements of small scale B-mode will measure the sum of the neutrino masses, which may solve the neutrino mass hierarchy problem. Experimental details of the PB-1 are found in ref. [3].

The Simons Array project is an upgrade plan of the PB-1 and will begin observation in 2016 at the Atacama plateau in Chile. The Simons Array has three newly designed receivers and three PB1 style telescopes. Before the full Simons Array project will start, the first new receiver on the one of the Simons Array telescopes, which we call POLARBEAR-2 (PB-2) project, will be deployed in 2015. Both PB-2 and Simons Array project are next generation experiments to characterize the inflationary and lensing B-mode signals. This paper will report the outline and instrumental details of the PB-2 project.

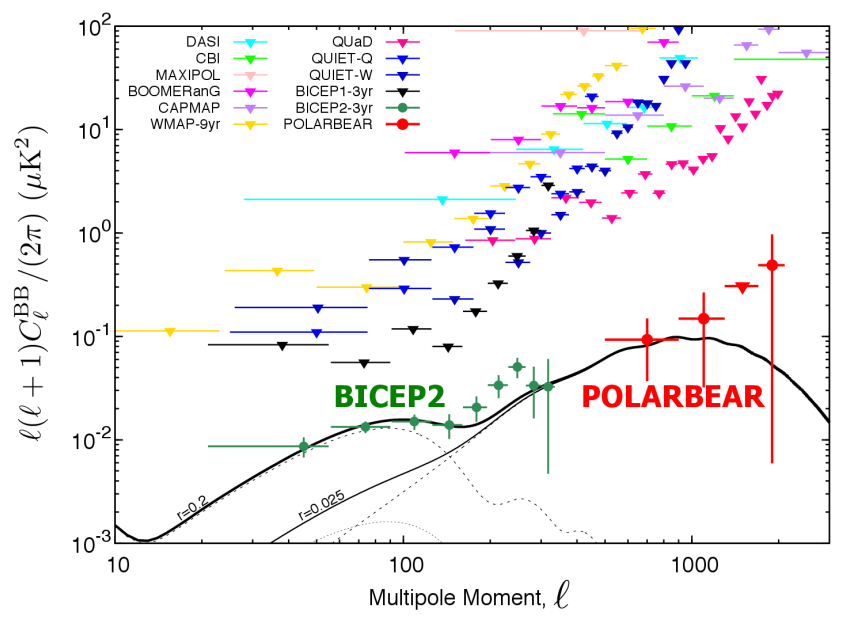

Figure 1: CMB B-mode polarization angular power spectrum measurements by previous experiments. 


\section{Project Overview}

Main features of the Simons Array project are: high sensitivity, high angular resolution ( 3.5 arcmin. at $150 \mathrm{GHz})$, simultaneous three band (90/150/220 GHz) observation, accessibility to a large fraction of sky (up to 80\%) and cost-efficient scalability. The Simons Array is the closet proposed experiment to the community consensus CMB Stage- 4 experiment. The ultimate goals about the inflation physics are:

- Tensor-to-scalar ratio $r$ measurement to $\sim 2 \%$ accuracy. Accessibility to large sky makes the sample variance limit as small as this accuracy level.

- Tensor index $n_{T}$ measurement with an error of 0.05 by using the de-lensing method. High angular resolution is beneficial to understand the lensing B-mode as a background for inflationary signals.

- Scalar index $n_{s}$ and its running $\alpha$ measurements with errors of 0.0015 and 0.0025 , respectively. The Simons Array can measure CMB E-mode polarization up to $l \sim 2000$ with good sensitivities thanks to the high sensitivity, high angular resolution and large sky coverage.

Figure 2 shows the Simons Array constraining power for both inflation and neutrino mass physics.
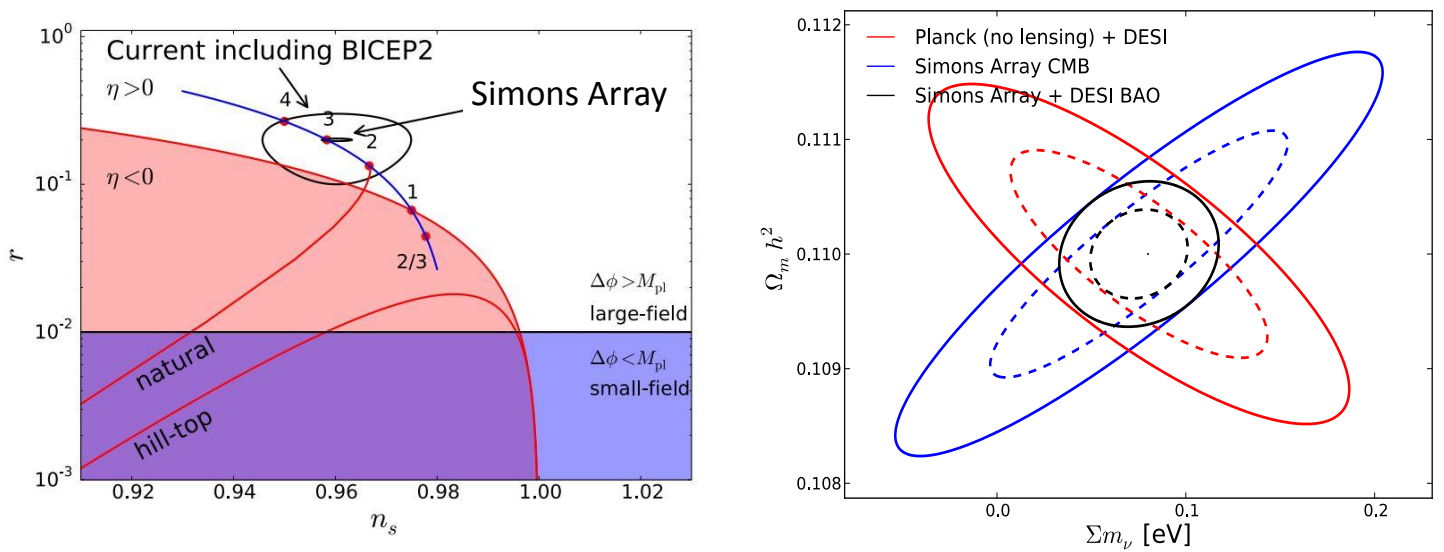

Figure 2: Left: Constraining power in $r$ vs. $n_{s}$ inflation parameter space by BICEP2 and Simons Array proposal. Black curves represent the $95 \%$ confidence-level regions. Right: The Simons Array constraints on the sum of the neutrino masses and matter density. Assumed theoretical value of the sum of the neutrino masses is $80 \mathrm{meV}$ in this plot. Combined with DESI BAO result, the degeneracy between these two parameters will be solved and the precision of $19 \mathrm{meV}$ on the sum of the neutrino masses will be achieved.

The PB-2 has a huge potential as a ground-based CMB polarization experiment on its own [4][5][6]. Table 1 shows the main specifications of the PB-2 project. The new PB-2 receiver will realize simultaneous dual-band observation at $95 \mathrm{GHz}$ and $150 \mathrm{GHz}$ [7]. The number of pixels are 6 times larger than the PB-1 receiver (The Simons Array has 3 times more pixels than the PB-2 project). They provide the total sensitivity (NET array) of $5.8 \mu \mathrm{K} \sqrt{s}$ for $95 \mathrm{GHz}$ and $150 \mathrm{GHz}$. Assuming 65\% sky coverage, $18 \%$ observation efficiency and 3 years observation, polarization sensitivity of the PB-2 project is $13.9 \mu \mathrm{K}$-arcmin. when $95 \mathrm{GHz}$ and $150 \mathrm{GHz}$ combined. Figure 
3 shows the expected sensitivities of the PB-2 B-mode observations in the case of tensor-to-scalar ratio of $\mathrm{r}=0.025$ and 0.2 , respectively. The high sensitivities of the PB-2 at $25<l<2500$ allow us to constrain the tensor-to-scalar ratio $\mathrm{r}$ by $2 \sigma$ level in case of $\mathrm{r}=0.01$ and the sum of neutrino masses by $1 \sigma$ level in case of $90 \mathrm{meV}$. The PB-2 is a very important step also for the demonstration of the full Simons Array advantages mentioned above.

\begin{tabular}{|c|c|}
\hline & Specifications \\
\hline Frequencies & $95 \mathrm{GHz}$ and $150 \mathrm{GHz}$ \\
\hline Number of Pixels & $1897(7588$ bolometers $)$ \\
\hline NET bolometer & $360 / 360 \mu \mathrm{K} \sqrt{s}$ for $95 / 150 \mathrm{GHz}$ \\
\hline NET array & $5.8 / 5.8 \mu \mathrm{K} \sqrt{s}$ for $95 / 150 \mathrm{GHz}$ \\
\hline Field of View & $4.8^{\circ}$ \\
\hline Beam Size & $5.2 / 3.5$ arcmin. for $95 / 150 \mathrm{GHz}$ \\
\hline
\end{tabular}

Table 1: The PB-2 receiver specifications.

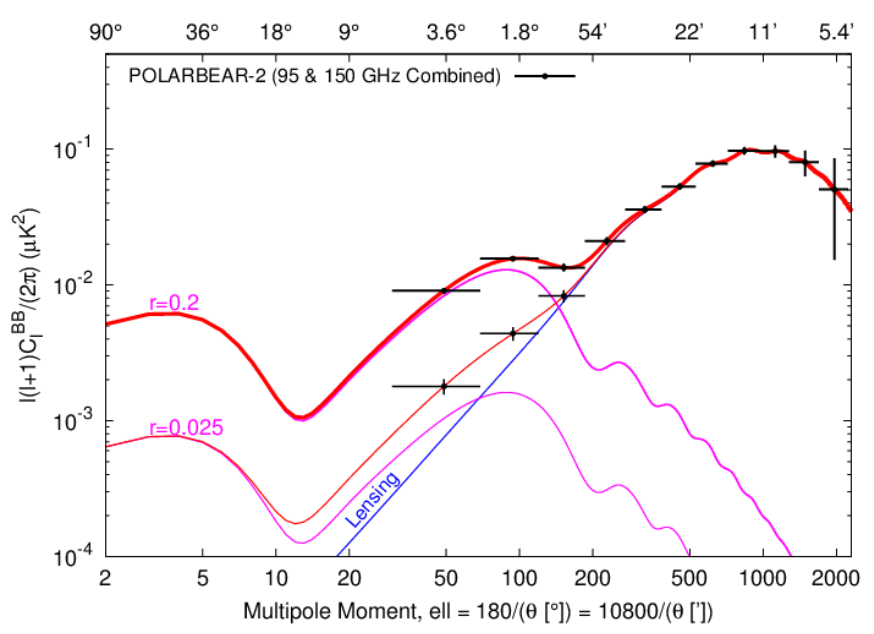

Figure 3: The PB-2 sensitivities on the CMB B-mode polarization angular power spectrum measurements.

\section{Receiver system}

PB-2 and Simons Array will use three identical Huan Tran Telescope (HTT) currently used in the PB-1 as shown in the left photo of Figure 4. The HTT is a 3.5 meter diameter off-axis Gregorian-Dragone telescope with low-sidelobe response, designed specifically for the CMB polarization measurement.

The right photo of Figure 4 shows the PB-2 receiver. The receiver size is $1.9 \mathrm{~m} \times 1.2 \mathrm{~m} \times$ $0.88 \mathrm{~m}$. It is similar to an ordinary single-lens reflex camera and has $4 \mathrm{~K}$ and $50 \mathrm{~K}$ nested shields inside 300K vacuum chamber. Two Pulse Tube Coolers (PTCs) are used for $4 \mathrm{~K}$ and $50 \mathrm{~K}$ cooling 
[5].

Figure 5 shows the schematic picture of the optical path in the PB-2 receiver. The light from the sky reflects at the primary and secondary mirrors and enters into the receiver through the 300 $\mathrm{K}$ window and 50K Infra-Red (IR) filter. Then, the light focuses onto the $250 \mathrm{mK}$ cooled focal plane by the $4 \mathrm{~K}$ cooled re-imaging three lenses. The focal plane is inside $4 \mathrm{~K}$ shield and cooled by the 3 stage helium sorption refrigerator. The focal plane has 1,897 lenslet-coupled pixels, each of which is dual-polarization and dual frequency with total of 7.588 Transition Edge Sensors (TES) bolometric detectors.
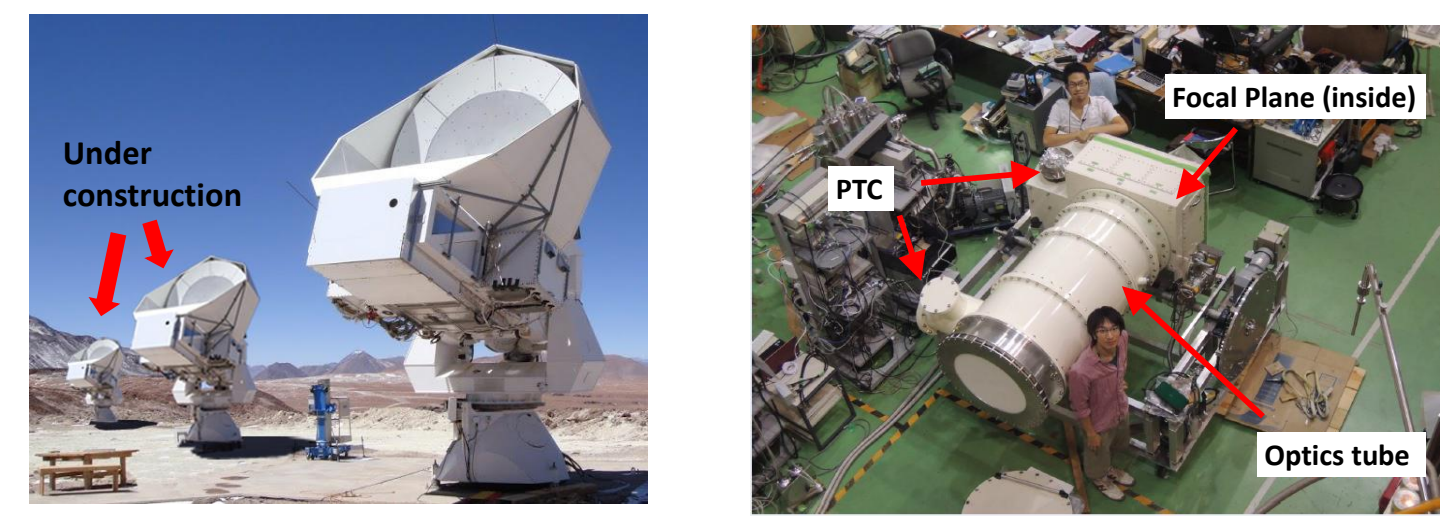

Figure 4: Left: One HHT for the PB-1 is located at Atacama plateau in Chile. Two more HHT is now under construction. Right: The PB-2 new receiver system.

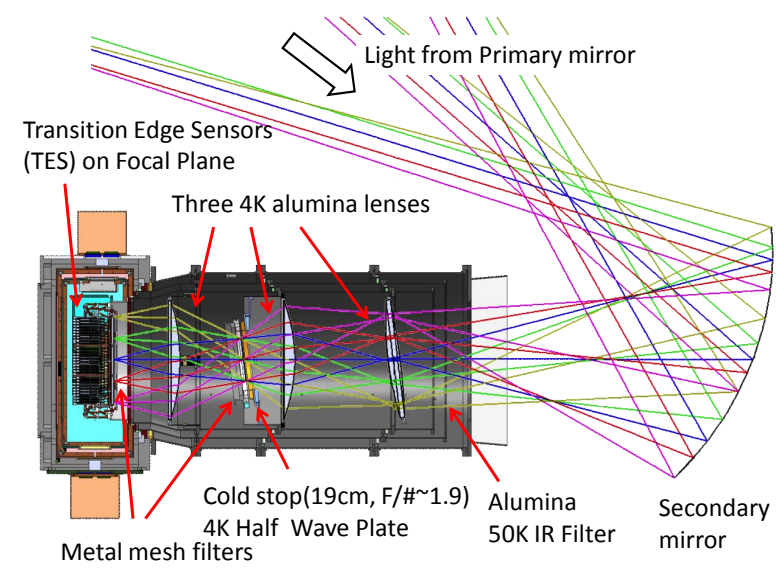

Figure 5: Schematic picture of the optical path in the PB-2 receiver system.

\subsection{Cold Optics}

To achieve the larger field of view $\left(4.8^{\circ}\right)$ than the PB-1's with high Strehl ratio, high purity Alumina (purity $>99.5 \%$ ) is used for three 50 -cm diameter re-imaging lenses because its index of refraction is relatively high $(\sim 3.1)$. Re-imaging makes the focus telecentric over the whole $35-\mathrm{cm}$ diameter focal plane. The loss tangent of Alumina for mm-meter waves is small $\sim 10^{-4}$ while 
Alumina absorbs IR light effectively. Therefore, the lenses also serve as IR filters at $4 \mathrm{~K}$ stage.

The main IR filter of the PB-2 receiver is placed at 50K stage. It is made by high purity Alumina (>99.9\%). Main features of the Alumina 50K IR filter is relatively low filter temperature thanks to its high thermal conductivity $(\sim 144 \mathrm{~W} / \mathrm{m} \cdot \mathrm{K}$ at $77 \mathrm{~K})$, which reduces the extra optical loading from filter itself [8].

As well as Alumina IR filter and lenses, metal mesh filters developed by Cardiff Univ. are used at $350 \mathrm{mK}$ and $4 \mathrm{~K}$ stages for the further thermal filters [9]. Their cut-off frequencies are just above the science bands we measure. Details of the optical elements are also found in ref. [4].

\subsection{Detectors and Focal Plane}

\section{$250 \mathrm{mK}$ focal plane}

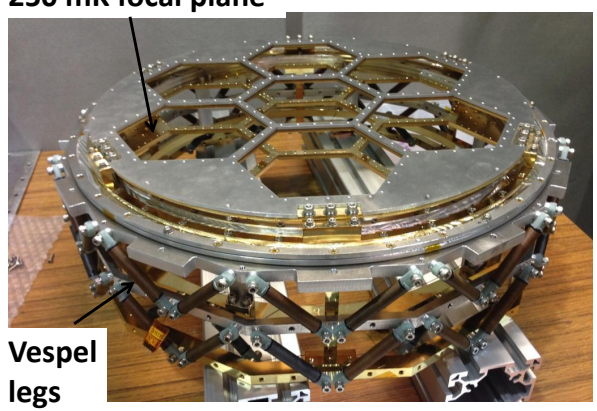

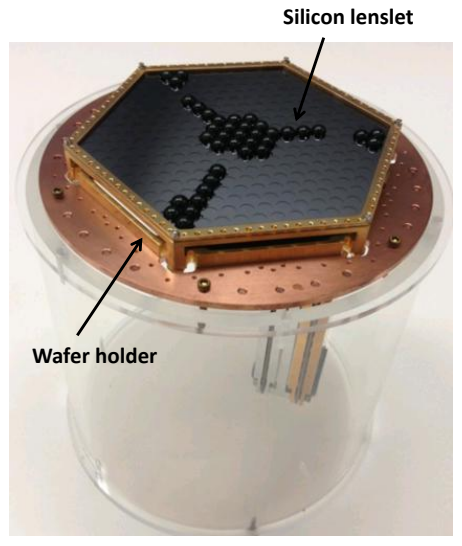

Figure 6: Left: The PB-2 focal plane tower without detector array modules. Right: The detector array module with some lenslets.
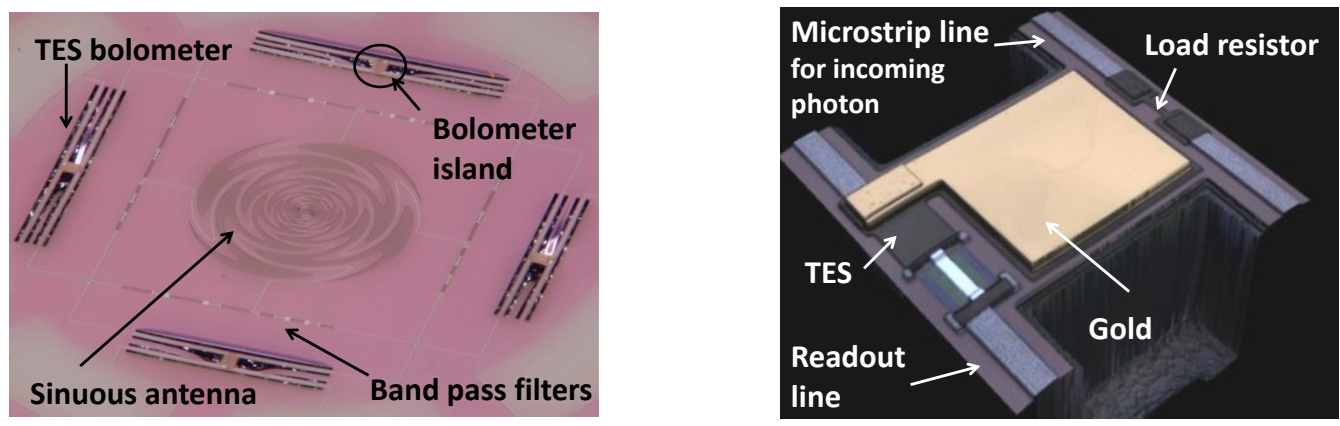

Figure 7: Left: One pixel on the detector wafer. Right: Zoomed 3-D microscope photograph of the bolometer island.

The left photo of Figure 6 shows the focal plane tower. The tower has $250 \mathrm{mK}$ focal plane, 350 $\mathrm{mK}, 2 \mathrm{~K}$ and $4 \mathrm{~K}$ stages. They are mechanically supported by vespel legs. A thermal conductivity of vespel legs is low enough. The former three stages are cooled by the 3 stage helium sorption refrigerator whose cooling power at $250 \mathrm{mK}$ stage is $10 \mu \mathrm{W}$. The $250 \mathrm{mK}$ focal plane is divided into seven detector array modules, which have 271 pixels. The right photo of Figure 6 shows one detector array module. It consists of $150 \mathrm{~mm}$ detector wafer, its holder made by Invar and readout 
components under the detector wafer (not shown in this photo).

Each pixel consists of a silicon lenslet, a log-periodic broadband sinuous antenna for dual polarization and dual frequency, a diplexing filter to split the incoming radiation into two bands and four TES bolometers. The silicon lenslet is hemispherical shape. Its surface is coated by twolayer epoxy for the anti-reflection [10]. One pixel details on the detector wafer are shown in the left photo of Figure 7. Bandwidth of the log-periodic broadband sinuous antenna is decided by the inner and outer radius of the antenna. Diplexing filters on the micro-strip line between the antenna and bolometers define a roughly 4:1 bandwidth for 95 and $150 \mathrm{GHz}$.

TES is a superconducting bolometric detector and sits on the silicon wafer island as shown in the left photo of Figure 7. The right photo of Figure 7 is a zoom and shows the details in the bolometer island. The incoming photon through the micro-strip line is converted into thermal signals by the load resistor on the bolometer island, then TES bolometer measures this thermal signal. A gold layer is added on the bolometer island for extra heat capacity. This makes the TES response time slow $(\sim 0.25 \mathrm{msec})$ enough to readout.

Detector array are mainly developed by UC. Berkeely and UC. San Diego. Details of detectors and their evaluated performance are also found in ref. [7].

\subsection{Readout}

Figure 8 shows the schematic drawing of a readout chain. It is a digital frequency domain multiplexing readout with superconducting quantum interference devise (SQUID) array amplifiers [11]. Each TES bolometer is AC voltage-biased and an analog sum of the AC currents from all TES bolometers is readout. A frequency of the AC current through each bolometer is decided by the LC filter in series with the bolometer. The room temperature readout board (DfMux board) provides the AC voltage-bias on the bolometers. At the same time, analog summed signals are demodulated by frequencies of each AC voltage-bias in the DfMux board. Another important technology we use with the DfMux board is a Digital Active Nulling (DAN) to increase the bandwidth of the SQUID electronics and to reduce the effective input impedance of the SQUID.[12].

We are also developing lithographed inductors and capacitors, niobium-titanium stripline cables and other readout components to achieve about 40 multiplexing factor readout using up to 3 $\mathrm{MHz}$ AC voltage-bias [13]. Lithographed inductors and capacitors are fabricated on a high resistivity $(>10 \mathrm{~K} \Omega / \mathrm{cm})$ silicon wafer to reduce the parasitic resistance. The niobium-titanium stripline cable is designed for the low thermal loading into the $\mathrm{mK}$ stages and the low stray inductance. To meet requirements on the crosstalk, the stray inductance should be $<50 \mathrm{nH}$. A measured inductance of test version cables is below this value.

\section{Conclusions}

PB-2 and Simons Array project are next generation ground-based CMB polarization experiments for the cosmology and high energy physics, especially inflation and neutrino physics. We have been developing new technologies such as broadband optical elements, broadband TES detectors and readout electronics while we fully make use of the cost-efficient scalability of the PB-1 experiment. 


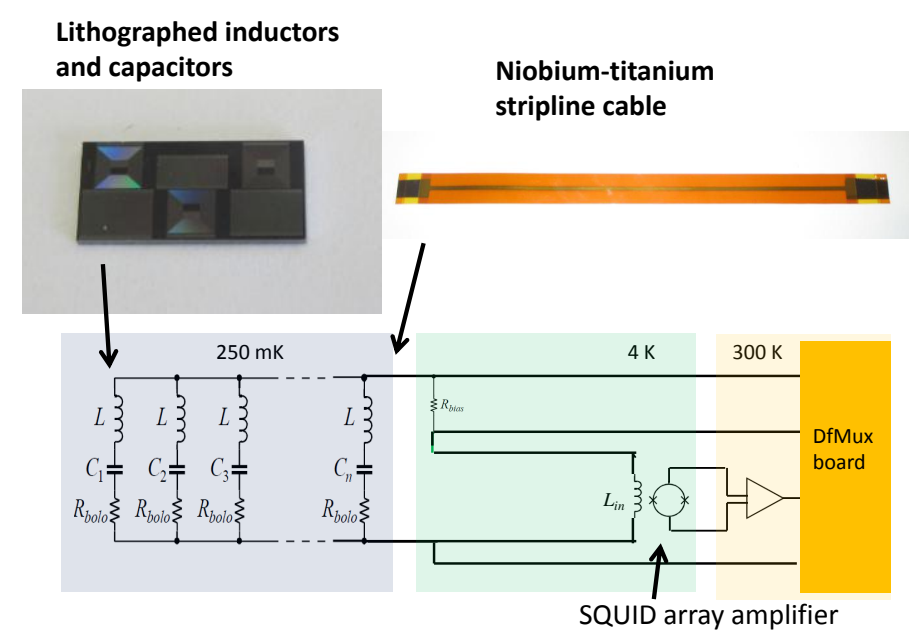

Figure 8: Readout schematic picture and photos of newly designed lithographed inductors and capacitors and niobium-titanium stripline cable.

\section{Acknowledgements}

The POLARBEAR-2 project is supported by the MEXR Kakenhi grant 21111002, NSF grant AST-0618398, NASA grant NNG06GJ08G, the Simons Foundation, Natural Sciences and Enginnering Research Council, Canadian Institute for Advanced Research and the CONICYT.

\section{References}

[1] The BICEP2 Collaboration, Phys. Rev. Lett. 112, 241101, 2014

[2] The POLARBEAR Collaboration, arXiv:1403.2369 [astro-ph.CO]

[3] K. Arnold et al., Proc.SPIE 8452 (2012) 84521D

[4] T. Matsumura et al., Proc.SPIE 8452 (2012) 84523E

[5] T. Tomaru et al., Proc.SPIE 8452 (2012) 84521H

[6] A. Suzuki et al., J Low Temp Phys, DOI 10.1007/s10909-014-1112-x (2014)

[7] A. Suzuki et al., Proc.SPIE 8452 (2012)

[8] Y. Inoue et al., Applied Optics Vol. 53, pp. 1727-1733 (2014)

[9] P. A. R. Ade et al., Proc.SPIE 6275 (2006) 62750U

[10] D. Rosen et al., Applied Optics Vol. 52, pp. $8102-8105$ (2013)

[11] M. A. Dobbs et al., Rev. Sci. Instrum. 83 (2012) 073113

[12] T. de Haan et al., Proc.SPIE 8452 (2012) 84520E

[13] K. Hattori et al., Nucl.Instrum.Meth. A732 (2013) 299-302 